\title{
Heterogeneously integrated III-V/silicon distributed feedback lasers
}

\author{
S. Keyvaninia, ${ }^{(1,2)}$ S. Verstuyft, ${ }^{(1,2)}$ L. Van Landschoot, ${ }^{(1,2)}$ F. Lelarge ${ }^{3}$ G. $-H$. Duan, ${ }^{3}$ S. \\ Messaoudene, ${ }^{4}$ J.M. Fedeli, ${ }^{4}$ T. De Vries, ${ }^{5}$ B. Smalbrugge, ${ }^{5}$ E.J.Geluk, ${ }^{5}$ J. Bolk, ${ }^{5}$ M. Smit, ${ }^{5}$ D. \\ Van Thourhout, ${ }^{(1,2)}$ G. Roelkens ${ }^{(1,2,5)}$ \\ ${ }^{1}$ Photonic Research Group, INTEC, Ghent University-IMEC, Sint-Pietersnieuwstraat 41, B-9000 Ghent. \\ ${ }^{2}$ Center for Nano- and Biophotonics (NB-Photonics), Ghent, Belgium \\ ${ }^{3}$ III-V Lab, a joint lab of 'Alcatel-Lucent Bell Labs France', 'Thales Research and Technology' and 'CEA Leti', Campus \\ Polytechnique, 1, Avenue A. Fresnel, 91767 Palaiseau cedex, France. \\ ${ }^{4}$ CEA Leti', LETI, Minatec, 17 rue des Martyrs, F-38054 GRENOBLE cedex 9, France. \\ ${ }^{5}$ Photonic Integration group, Eindhoven University of Technology, Den Dolech 2, Eindhoven, The Netherlands. \\ *Corresponding author: Shahram.Keyvaninia@intec.ugent.be
}

Received Month X, XXXX; revised Month X, XXXX; accepted Month X, XXXX; posted Month X, XXXX (Doc. ID XXXXX); published Month X, XXXX

Heterogeneously integrated III-V-on-silicon second order distributed feedback lasers utilizing an ultra-thin DVS-BCB die-to-wafer bonding process are reported. A novel design exploiting high confinement in the active waveguide is demonstrated. $14 \mathrm{~mW}$ output power coupled to a silicon waveguide, $50 \mathrm{~dB}$ side mode suppression ratio and continuous wave operation up to $60^{\circ} \mathrm{C}$ is obtained.@ 2013 Optical Society of America OCIS Codes: (130.0250) Optoelectronics, (250.5300) Photonic integrated circuits, (140.3490) Lasers, distributed-feedback, (060.2420). http://dx.doi.org/10.1364/OL.99.099999

Silicon photonics is emerging as an important platform for the realization of high-speed optical transceivers. This is related to the fact that the silicon waveguide circuits, comprising ultra-compact passive waveguide circuitry, high-speed optical modulators and germanium photodetectors, can be fabricated using complementary metal-oxide-semiconductor (CMOS) fabrication technology in large volumes and at low cost [1]. However, the integration of a coherent light source on the silicon platform remains an issue. While electrically driven germanium laser sources have been demonstrated [2], the performance of these devices is still far inferior to what can be achieved using InP-based III-V semiconductors. III-V semiconductor layer stacks can be heterogeneously integrated onto the silicon waveguide circuit using a wafer bonding technique followed by InP substrate removal, which provides a route towards wafer-scale processing of these III-V epitaxial layers, lithographically aligned to the underlying waveguide circuit. In recent years, several device demonstrations were made on this III-V/silicon platform [3], both using a molecular [4] and adhesive bonding approach [5]. In this paper we describe the realization of single wavelength $1550 \mathrm{~nm}$ distributed feedback (DFB) lasers coupled to a $220 \mathrm{~nm}$ thick silicon waveguide layer, with waveguide coupled output powers of $14 \mathrm{~mW}$, a side-mode-suppression ratio better than $50 \mathrm{~dB}$ and a laser linewidth of $1 \mathrm{MHz}$. The coupling to a $220 \mathrm{~nm}$ silicon waveguide circuit will allow in a later stage to co- $^{-}$ integrate high speed devices such as modulators and photodetectors with the single wavelength lasers using the available silicon photonics platform technology as offered by several multi-project wafer run services worldwide.

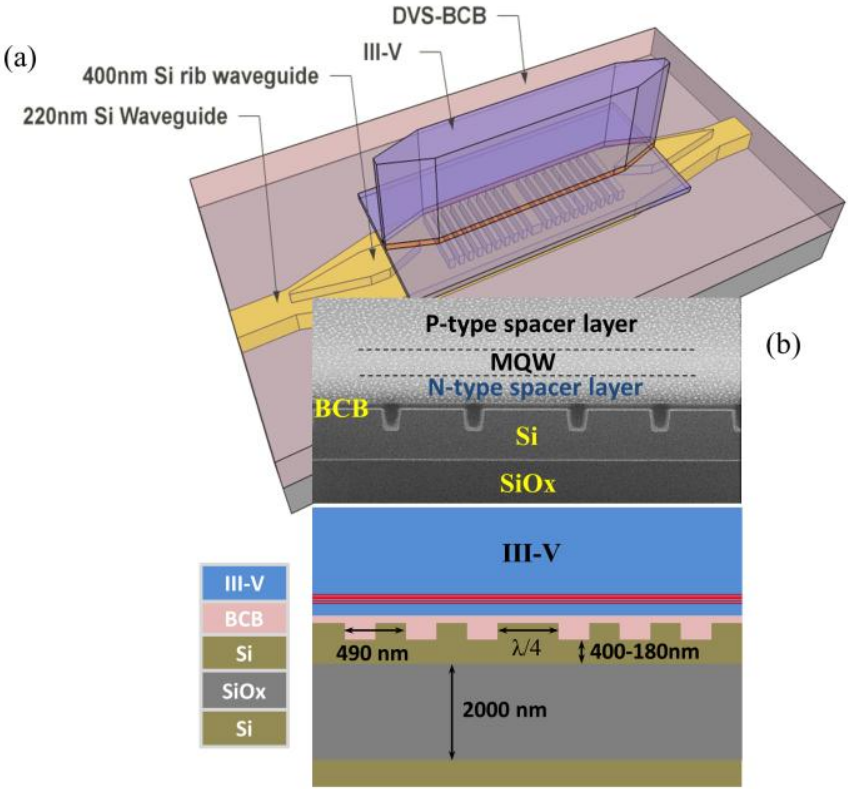

Fig.1. III-V/Si distributed feedback laser design: (a) threedimensional view on the III-V-on-silicon DFB laser; (b) schematic longitudinal cross-section of the laser structure and SEM picture of the actual device.

The distributed feedback laser structures reported in this paper are based on quarter-wave shifted second order gratings with a Bragg wavelength around $1550 \mathrm{~nm}$. The three dimensional layout of the laser cavity is depicted in Figure 1(a), while a longitudinal cross-section of the laser geometry is shown in Figure 1(b). The gratings are 
defined in a $400 \mathrm{~nm}$ thick silicon waveguide layer, by a $180 \mathrm{~nm}$ deep dry etch. The III-V epitaxial layer stack that is used consists of an $200 \mathrm{~nm}$ thick $\mathrm{n}$-InP contact layer, two $100 \mathrm{~nm}$ thick InGaAsP separate confinement heterostructure layers (bandgap wavelength 1.17um), 6 InGaAsP quantum wells (6nm thick, emission wavelength $1.55 \mu \mathrm{m}$ ) surrounded by InGaAsP barriers, a $1.5 \mu \mathrm{m}$ thick $\mathrm{p}-\mathrm{InP}$ top cladding and a $100 \mathrm{~nm} \mathrm{p}++\mathrm{InGaAs}$ contact layer. The confinement factor of the optical mode in the 6 quantum wells is $9.3 \%$. The use of a $400 \mathrm{~nm}$ thick silicon waveguide layer in the laser section simplifies the optical coupling between the III-V laser mode, which is completely confined in the III-V mesa and which evanescently feels the silicon grating, and the silicon waveguide layer, compared to the case of direct coupling to a $220 \mathrm{~nm}$ thick device layer. This optical coupling is realized using a III-V/silicon spotsize converter structure defined by tapering both the $\mathrm{II}-\mathrm{V}$ and silicon waveguide as shown in Figure 2(a), similar to the structure used in [6]. The III-V taper is a piecewise linear taper that quickly tapers $(\mathrm{L}=35 \mu \mathrm{m})$ from a $3 \mu \mathrm{m}$ mesa width to an $0.9 \mu \mathrm{m}$ wide waveguide width after which a slower adiabatic taper $(\mathrm{L}=150 \mu \mathrm{m})$ is implemented by tapering both the III-V and silicon waveguide structure. Using a $400 \mathrm{~nm}$ thick silicon waveguide relaxes the requirements on the required III-V taper tip width: a width of $500 \mathrm{~nm}$ is sufficient to achieve a high efficiency, low reflection power transfer from the III-V laser mesa to the silicon waveguide as illustrated by the simulation results in Figure 2(b). Since the III-V spotsize converter consists of the same active region as the laser, also this spot-size converter is electrically pumped. After the laser emission is coupled to the $400 \mathrm{~nm}$ thick silicon waveguide layer, a second spot-size converter $(\mathrm{L}=30 \mu \mathrm{m})$ is used to couple to a $220 \mathrm{~nm}$ strip waveguide. Quarter-wave shifted second order DFB gratings with a period of $480 \mathrm{~nm}$ and $490 \mathrm{~nm}$ and a duty cycle of $25 \%$ were studied. Since the evanescent tail of the laser mode is interacting with the silicon grating, the actual grating coupling strength depends on the DVS-BCB low index $(n=1.54)$ bonding layer thickness in between the III-V mesa and the silicon waveguide layer. This coupling strength is illustrated in Figure 2(c). Given the good uniformity and reproducibility of the adhesive bonding process [7], good control of the grating coupling strength can be obtained.

The silicon device wafer fabrication is carried out in a CMOS pilot line on 8inch SOI wafers. The process starts with the etching of a $400 \mathrm{~nm}$ silicon device layer on a $2 \mu \mathrm{m}$ $\mathrm{SiO}_{2}$ buried oxide layer. In a first etch step $180 \mathrm{~nm}$ deep gratings are etched together with the silicon rib waveguides used in the spotsize converter structure. In a second etch step, $50 \mathrm{~nm}$ deep structures are etched in the exposed $220 \mathrm{~nm}$ thick silicon device layer in order to realize fiber-to chip grating couplers.
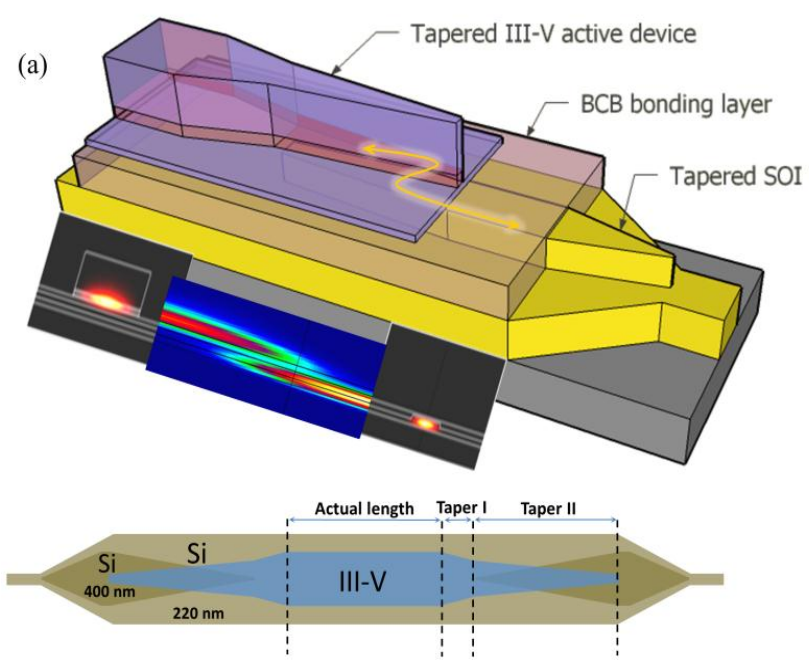

(b)
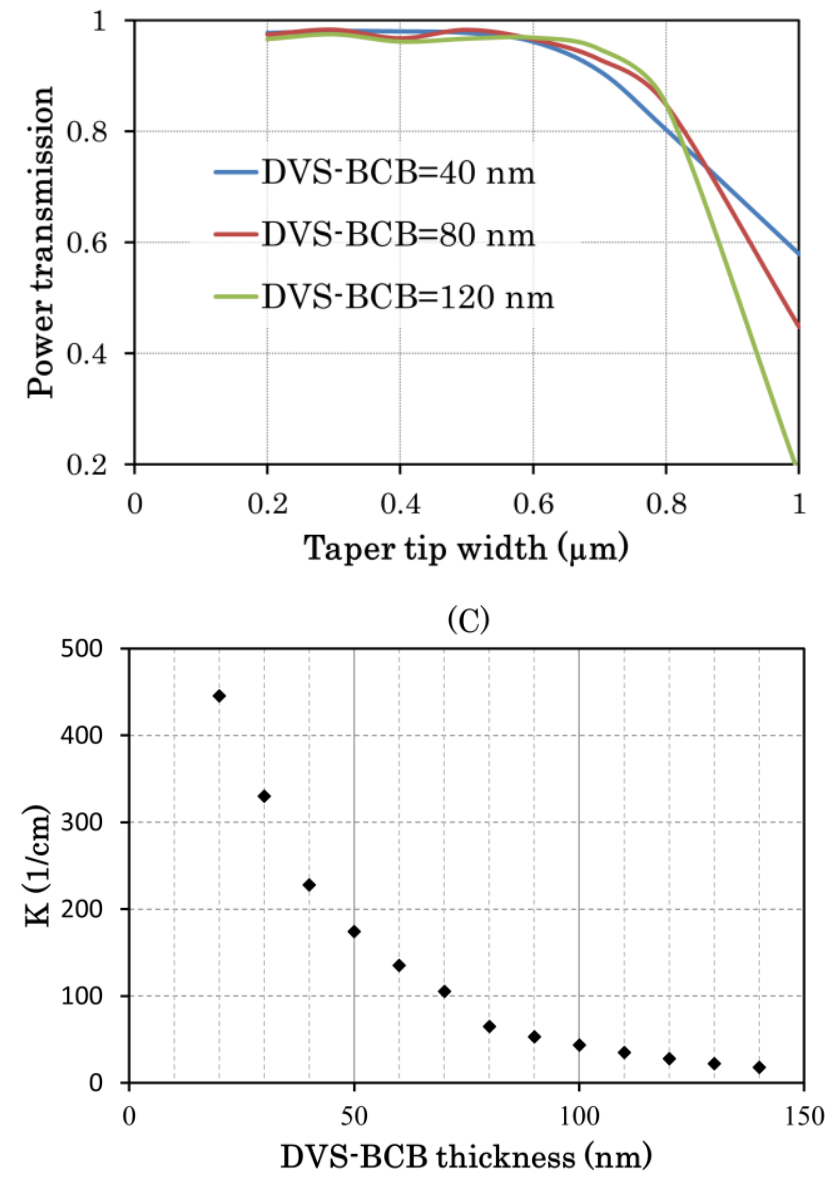

Fig. 2. (a) spotsize converter structure used in this work; (b) simulated power transmission and reflection at the spotsize converter taper tip as a function of taper tip width; (c) grating coupling strength as a function of DVS-BCB thickness for the second order grating structure $(490 \mathrm{~nm}$ period, $25 \%$ duty cycle) used in this work. 


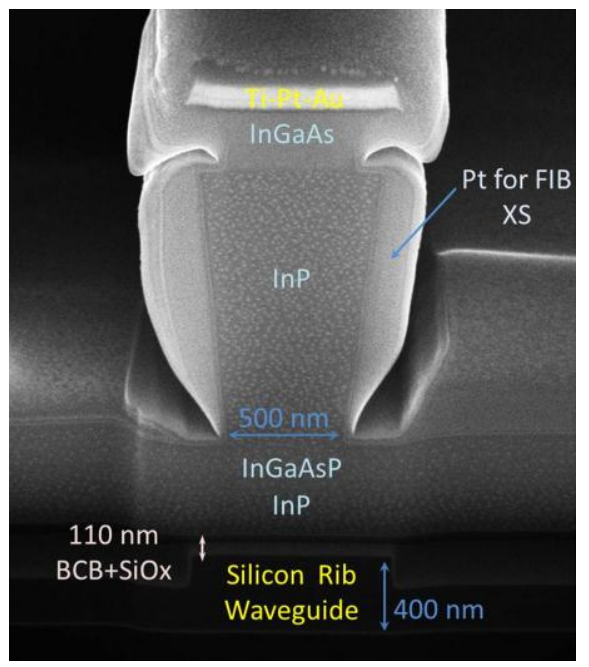

Fig. 3. . Scanning electron microscope images of the III-V spot-size converter taper tip illustrating the 500nm taper tip width close to the active core of the III-V waveguide.

In a final step a $220 \mathrm{~nm}$ deep etch is used to define the silicon strip waveguides and the transition to the 400nm device layer. After device etching, the wafer is covered with thick $\mathrm{SiO}_{2}$ after which chemical mechanical polishing is used to planarize the wafer in combination with wet chemical etching in buffered HF to expose the top of the $400 \mathrm{~nm}$ thick silicon waveguides. This way, a quasi-planar wafer surface is obtained, which allows the subsequent heterogeneous integration of the III-V epitaxial layer stack. For the transfer of the laser epitaxy, an adhesive die-to-wafer bonding process with a DVS-BCB adhesive bonding layer is used. This bonding process is described in detail in [7]. The 110nm bonding layer thickness results in a theoretical grating coupling strength of $35 \mathrm{~cm}^{-1}$. After bonding, the InP growth substrate is removed and the III$\mathrm{V}$ laser mesa is defined. In this device demonstration the III-V mesa and spotsize converter are defined through wet etching, using $\mathrm{HCl}$ and $\mathrm{H}_{2} \mathrm{O}: \mathrm{H}_{2} \mathrm{SO}_{4}: \mathrm{H}_{2} \mathrm{O}_{2}$ for the $\mathrm{InP}$ and InGaAs/InGaAsP layers respectively. This wet etching technique allowed creating undercut structures in the spot-size converter, which again relaxed the lithography requirements in the definition of the III-V spotsize converter, which was realized using $300 \mathrm{~nm}$ UV contact lithography. A scanning electron microscope picture of the $\mathrm{V}$-shaped III-V spotsize converter taper tip is shown in Figure 3.

Laser characterization was carried out by collecting the laser emission in a standard single mode fiber using integrated silicon fiber-to-chip grating coupler structures. The coupling efficiency of these grating couplers was characterized on separate structures and used to determine the optical power coupled to the $220 \mathrm{~nm}$ silicon strip waveguides. The L-I-V curves of the fabricated laser structures were measured both at room temperature and at elevated temperature. The results for a second order grating DFB laser (with a period of $490 \mathrm{~nm}$ and a device length - not including the spotsize converter - of $680 \mathrm{\mu m}$ ) are shown in Figure 4 . At $20^{\circ} \mathrm{C}$ and a $160 \mathrm{~mW}$ drive

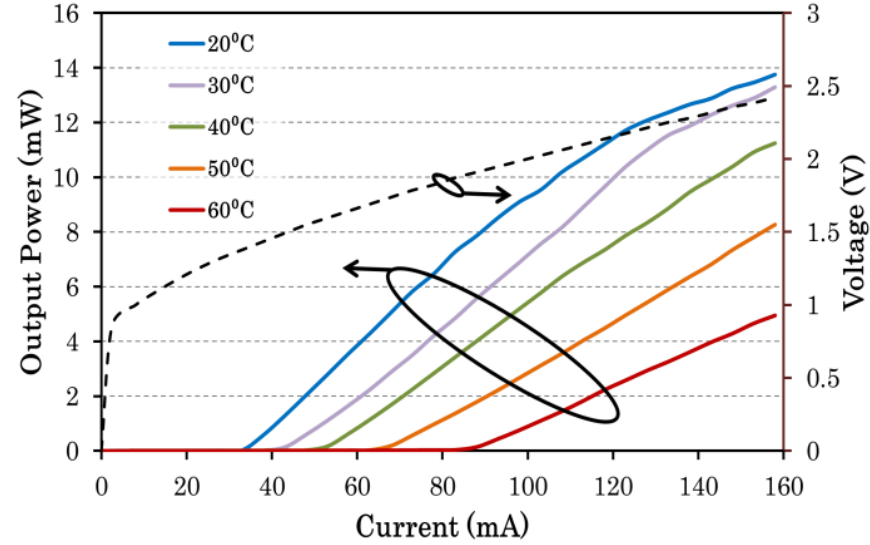

Fig. 4. L-I-V curves at different temperatures for a $490 \mathrm{~nm}$ period second order DFB laser.

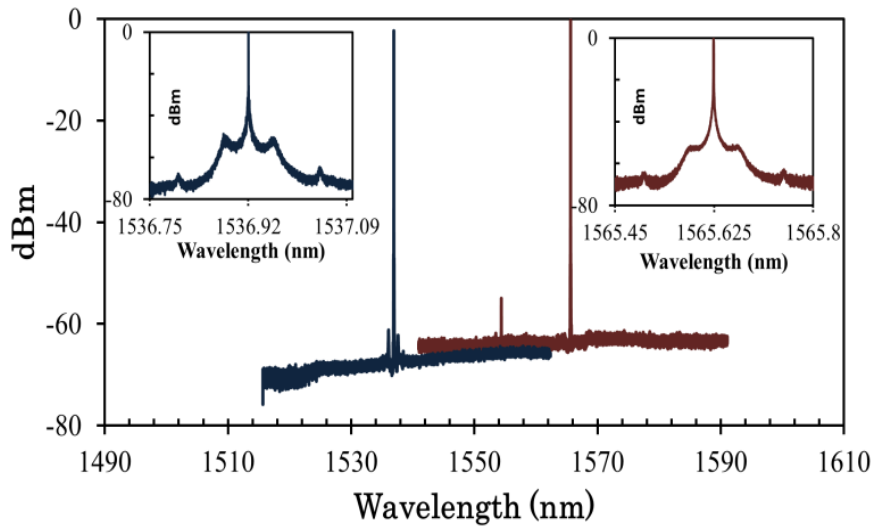

Fig. 5. Spectral characteristics of two second order DFB lasers (480nm period and 490nm period), illustrating single mode emission. The insets show the high-resolution spectra.

current an optical output power of $14 \mathrm{~mW}$ coupled into the silicon waveguide is obtained, with a threshold current of $35 \mathrm{~mA}$. The characteristic temperature $\mathrm{T}_{0}$ of the laser is 80 $\mathrm{K}$. The slope efficiency of the laser is $0.135 \mathrm{~W} / \mathrm{A}$ at $20^{\circ} \mathrm{C}$. At $60^{\circ} \mathrm{C}$ still $4 \mathrm{~mW}$ optical output power is obtained. The series resistance of the laser was 7.5 2 . Figure 5(a) and 5(b) show the laser spectrum for two second order DFB lasers with a grating period of $480 \mathrm{~nm}$ and $490 \mathrm{~nm}$ respectively (both a broad wavelength scan and a narrow wavelength scan, measured with a $15 \mathrm{MHz}$ resolution spectrum analyzer), showing clearly single mode operation with a side mode suppression ratio of more than 50dB. Single mode operation was observed over the whole laser drive current range, indicating that no significant spatial hole burning occurs. Finally, the laser linewidth was characterized using a delayed self-heterodyne measurement setup, using $22.5 \mathrm{~km}$ of optical fiber delay and an $80 \mathrm{MHz}$ acousto-optic modulator. These measurements indicate a laser linewidth of $1 \mathrm{MHz}$.

In conclusion, heterogeneous III-V/silicon DFB lasers with high optical confinement in the III-V laser mesa coupled to a silicon strip waveguide circuit were realized. The good device performance allows its use both for short-distance and long-distance optical communication. This demonstration paves the way for the realization of advanced III-V/silicon transmitter modules comprising 
III-V/silicon DFB lasers, silicon optical modulators and Ge photodetectors.

Acknowledgements:

Support of the EU commission through the EU-project HELIOS .

\section{Reference}

1. S. Selvaraja, W. Bogaerts, P. Dumon, D. Van Thourhout, R. Baets, IEEE Journal on Selected Topics in Quantum Electronics, 16(1), p.316 - 324 (2010)

2. Rodolfo E. Camacho-Aguilera, Yan Cai, Neil Patel, Jonathan T. Bessette, Marco Romagnoli, Lionel C. Kimerling, and Jurgen Michel, Opt. Express, Vol. 20, Issue 10, pp. 11316-11320 (2012)

3. G. Roelkens, L. Liu, D. Liang, R. Jones, A. Fang, B. Koch, J. Bowers, Laser Photonics Rev. 4(6) p. $751-$ 779 (2010).

4. H. Park, A. W. Fang, O. Cohen, R. Jones, M. J. Paniccia, and J. E. Bowers, IEEE Photon. Technol. Lett. 19, 230-232 (2007).

5. S. Keyvaninia, G. Roelkens, Dries Van Thourhout, G. Duan, M. Lamponi, F. Lelarge, J-M. Fedeli, S. Messaoudene, E. J. Geluk, and B. Smalbrugge, Proc. IEEE Group IV Photonics Conf., San Diego, United Sates, Sep. (2012)

6. A. W. Fang, H. Park, O. Cohen, R. Jones, M. J. Paniccia, and J. E. Bowers, Opt. Express 14, 92039210 (2006).

7. S. Keyvaninia, G. Roelkens, D. Van Thourhout, C. Jany, M. Lamponi, A. Le Leipvre, F. Lelarge, D. Make, G. Duan, D. Bordel, J.M. Fedeli, Opt. Express 21, 2784-3792 (2013).

8. S. Keyvaninia, M. Muneeb, S. Stankovic, René van Veldhoven, D. Van Thourhout, G. Roelkens, Optical Materials Express, Vol. 3, Issue 1, pp. 35-46 (2013) 
Reference

[1] S. Selvaraja, W. Bogaerts, P. Dumon, D. Van Thourhout, R. Baets, "Sub-nanometer linewidth uniformity in silicon nano-photonic waveguide devices using CMOS fabrication technology ," IEEE Journal on Selected Topics in Quantum Electronics, 16(1), p.316 - 324 (2010)

[2] Rodolfo E. Camacho-Aguilera, Yan Cai, Neil Patel, Jonathan T. Bessette, Marco Romagnoli, Lionel C. Kimerling, and Jurgen Michel, "An electrically pumped germanium laser," Optics Express, Vol. 20, Issue 10, pp. 11316-11320 (2012)

[3] G. Roelkens, L. Liu, D. Liang, R. Jones, A. Fang, B. Koch, J. Bowers, "III-V/silicon photonics for on-chip and inter-chip optical interconnects," Laser Photonics Rev. 4(6) p. 751 - 779 (2010).

[4] H. Park, A. W. Fang, O. Cohen, R. Jones, M. J. Paniccia, and J. E. Bowers, "An electrically pumped AlGaInAs-Silicon Evanescent Amplifier," IEEE Photon. Technol. Lett. 19, 230232 (2007).

[5] S. Keyvaninia, G. Roelkens, Dries Van Thourhout, G. Duan, M. Lamponi, F. Lelarge, JM. Fedeli, S. Messaoudene, E. J. Geluk, and B. Smalbrugge, "A highly efficient electrically pumped optical amplifier integrated on a SOI waveguide circuit," in Proc. IEEE Group IV Photonics Conf., San Diego, United Sates, Sep. (2012)

[6] A. W. Fang, H. Park, O. Cohen, R. Jones, M. J. Paniccia, and J. E. Bowers, "Electrically pumped hybrid AlGaInAs-silicon evanescent laser," Opt. Express 14, 9203-9210 (2006).

[7] S. Keyvaninia, G. Roelkens, D. Van Thourhout, C. Jany, M. Lamponi, A. Le Leipvre, F. Lelarge, D. Make, G. Duan, D. Bordel, J.M. Fedeli, "Demonstration of a heterogeneously integrated III-V/SOI single wavelength tunable laser," Opt. Express 21, 2784-3792 (2013).

[8] S. Keyvaninia, M. Muneeb, S. Stankovic, René van Veldhoven, D. Van Thourhout, G. Roelkens, Ultra-thin DVS-BCB adhesive bonding of III-V wafers, dies and multiple dies to a patterned silicon-on-insulator substrate, Optical Materials Express, Vol. 3, Issue 1, pp. 35-46 (2013) 